\title{
ON THE USE OF SPECTRAL MINUTIAE IN HIGH-RESOLUTION PALMPRINT RECOGNITION
}

\author{
Ruifang Wang ${ }^{1}$, Raymond Veldhuis ${ }^{2}$, Daniel Ramos ${ }^{1}$, Luuk Spreeuwers ${ }^{2}$, Julian Fierrez ${ }^{1}$, Haiyun Xu ${ }^{3}$ \\ ${ }^{1}$ Biometric Recognition Group-ATVS, EPS, Universidad Autonoma de Madrid, Spain \\ ${ }^{2}$ Signals and Systems Group, EEMCS, University of Twente, the Netherlands \\ ${ }^{3}$ Software Improvement Group B.V., Amsterdam, the Netherlands
}

\begin{abstract}
The spectral minutiae representation has been proposed as a novel method to minutiae-based fingerprint recognition, which can handle minutiae translation and rotation and improve matching speed. As high-resolution palmprint recognition is also mainly based on minutiae sets, we apply spectral minutiae representation to palmprints and implement spectral minutiae based matching. We optimize key parameters for the method by experimental study on the characteristics of spectral minutiae using both fingerprints and palmprints. However, experimental results show that spectral minutiae representation has much worse performance for palmprints than that for fingerprints. EER $15.89 \%$ and $14.2 \%$ are achieved on the public highresolution palmprint database THUPALMLAB using location-based spectral minutiae representation (SML) and the complex spectral minutiae representation (SMC) respectively while $5.1 \%$ and $3.05 \%$ on $\mathrm{FVC} 2002 \mathrm{DB} 2 \mathrm{~A}$ fingerprint database. Based on statistical analysis, we find the worse performance for palmprints mainly due to larger non-linear distortion and much larger number of minutiae.
\end{abstract}

Index Terms - Spectral minutiae, fingerprints, highresolution palmprints

\section{INTRODUCTION}

Recently, the spectral minutiae representation [1-2] has shown its power in minutiae-based fingerprint recognition, which can handle minutiae translation and rotation and improve matching speed, satisfying properties required by high-resolution palmprint recognition as well. As defined in [1], the method uses the minutiae locations in spatial domain and takes Fourier transform of the coded locations and obtains the magnitude of its Fourier spectrum in frequency domain. The three types of spectral minutiae representations are the location-based spectral minutiae representation (SML), the orientation-based spectral minutiae representation (SMO) and the complex spectral minutiae representation (SMC), among which the enhanced SMC method [2] performs best for fingerprints with the EER of $3.05 \%$ on FVC2002 DB2 $\mathrm{A}^{1}$ database and a matching speed of 8000 comparisons per second.
Inspired by matching accuracy and efficiency of spectral minutiae representation, we apply it to palmprints and implement spectral minutiae based matching as highresolution palmprint recognition is also mainly based on minutiae sets. In this work, we first optimize key parameters for the SML and SMC method by experimental study on the characteristics of spectral minutiae representation using both fingerprints and palmprints. Then we implement the direct matching as stated in [1-2] for palmprints and evaluate the performance of SML and SMC respectively on the public high-resolution palmprint database THUPALMLAB [3]. Results show that spectral minutiae representation has much worse performance in palmprints than in fingerprints. EER $15.89 \%$ and $14.2 \%$ are achieved on the palmprint database using SML and SMC respectively while $5.1 \%$ and $3.05 \%$ on FVC2002 DB2A ${ }^{1}$. Following a statistical analysis and comparison between palmprints and fingerprints, we find a worse performance for palmprints mainly due to larger nonlinear distortion and much larger number of minutiae.

The rest of this paper is organized as follows: Section 2 describes the spectral minutiae representation method. Section 3 describes experimental parameter optimization. Experimental results and discussion are provided in Section 4, with conclusions presented in Section 5.

\section{SPECTRAL MINUTIAE FOR HIGH-RESOLUTION FULL PALMPRINTS}

The spectral minutiae representation for high-resolution palmprints is the same with that for fingerprints. Note that we have summarized the parameters for the method in Table 1 to simplify the notations in (1) - (6). Given the minutiae set $\left\{\left(x_{i}, y_{i}, \theta_{i}\right)\right\}_{i=1}^{Z}$ containing $Z$ minutiae in a palmprint. Firstly, in spatial domain, the minutiae locations of a palmprint are coded by Gaussian indicator functions, $m\left(x, y ; \sigma^{2}\right)=$

$$
\sum_{i=1}^{Z} \frac{1}{2 \pi \sigma^{2}} \exp \left(-\frac{\left(x-x_{i}\right)^{2}+\left(y-y_{i}\right)^{2}}{2 \sigma^{2}}\right), \sigma=\sigma_{L} \text { or } \sigma_{C}
$$

Then in frequency domain, we take the Fourier transform of $m\left(x, y ; \sigma^{2}\right)$ and obtain the magnitude of Fourier spectrum, i.e., for SML representation,

$\mathcal{M}_{L}\left(\omega_{x}, \omega_{y} ; \sigma_{L}^{2}\right)=$

$$
\left|\exp \left(-\frac{\omega_{x}^{2}+\omega_{y}^{2}}{2 \sigma_{L}^{-2}}\right) \sum_{i=1}^{Z} \exp \left(-\mathrm{j}\left(\omega_{x} x_{i}+\omega_{\mathrm{y}} \mathrm{y}_{\mathrm{i}}\right)\right)\right|
$$

and for SMC representation, 


$$
\begin{aligned}
& \mathcal{M}_{C}\left(\omega_{x}, \omega_{y} ; \sigma_{C}^{2}\right)= \\
& \quad\left|\exp \left(-\frac{\omega_{x}^{2}+\omega_{y}^{2}}{2 \sigma_{C}^{-2}}\right) \sum_{i=1}^{Z} \exp \left(-\mathrm{j}\left(\omega_{x} x_{i}+\omega_{y} y_{i}\right)+\mathrm{j} \theta_{i}\right)\right| .
\end{aligned}
$$

Finally, the continuous spectra SML or SMC are sampled on a polar-linear grid with the size $M \times N$ where $M$ samples are located in the radial direction between $\lambda_{l}$ and $\lambda_{h}$, and $N$ samples are located in the angular direction betwem 0 and $\pi$ for SML or between 0 and $2 \pi$ for SMC. Especially, when $\sigma=0$, it means each minutia is presented by a Dirac pulse $m_{i}(x, y)=\delta\left(x-x_{i}, y-y_{i}\right)$, and the SML representation becomes

$$
\mathcal{M}_{L}\left(\omega_{x}, \omega_{y} ; \sigma_{L}^{2}\right)=\sum_{i=1}^{Z} \exp \left(-\mathrm{j}\left(\omega_{x} x_{i}+\omega_{\mathrm{y}} \mathrm{y}_{\mathrm{i}}\right)\right)
$$

and the SML representation becomes

$$
\mathcal{M}_{C}\left(\omega_{x}, \omega_{y} ; \sigma_{C}^{2}\right)=\sum_{i=1}^{Z} \exp \left(-\mathrm{j}\left(\omega_{x} x_{i}+\omega_{y} y_{i}\right)+\mathrm{j} \theta_{i}\right),
$$

Given $R(m, n)$ and $T(m, n)$ be the two sampled minutiae spectra, respectively, achieved from the reference palmprint and test palmprint, spectral minutiae matching score will be caculated by

$$
S^{(R, T)}=\max _{k}\left\{\frac{1}{M N} \sum_{m, n} R(m, n) T(m, n-k)\right\}, k \in[-128,128]
$$

Note that we use the full angular shift range $[-128,128]$ for palmprint matching in Section 4, while the smaller range $[-15,15]$ only for sample images in Section 3 , because the whole palmprint database we use have much lager rotation.

The parameters in (1) - (6) are described in Table 1. And parameter optimization will be discussed in Section 3.

Table 1. Description of parameters

\begin{tabular}{c|c|c}
\hline \hline Parameters & Values & Descriptions \\
\hline$M$ & 128 & Number of polar samples for radius \\
\hline$N$ & 256 & Number of polar samples for angle \\
\hline$\sigma_{L}$ & $>=0$ & $\begin{array}{c}\text { Gaussian parameter for SML } \\
\text { When 0, denotes Dirac; } \\
\text { otherwise, Gaussian }\end{array}$ \\
\hline$\sigma_{C}$ & $>=0$ & $\begin{array}{c}\text { Gaussian parameter for SMC } \\
\text { When 0, denotes Dirac pulse; } \\
\text { otherwise, Gaussian pulse }\end{array}$ \\
\hline$\lambda_{l}$ & $>=0$ & Lower bound of frequency range \\
\hline$\lambda_{h}$ & $>0$ & Upper bound of frequency range \\
\hline $\begin{array}{c}\text { Shift } \\
\text { Range } k\end{array}$ & $\begin{array}{c}{[-128,} \\
128]\end{array}$ & Setting for rotation compensation \\
\hline \hline
\end{tabular}

\section{PARAMETER OPTIMIZATION FOR SPECTRAL MINUTIAE}

As shown in Table 1, the Gaussian parameters $\sigma_{L}$ and $\sigma_{C}$ and the frequency range $\left[\lambda_{l}, \lambda_{h}\right]$ are to be optimized. In order to obtain optimal settings for the parameters, experimental study is executed as below. In this section, testing fingerprints are 1_1.bmp, 1_2.bmp, 2_1.bmp from FVC2002 DB2A, and testing palmprints are 80_1_1.bmp, 80 1 2.bmp, 80 r 1.bmp from the high-resolution palmprint database THUPALMLAB. And minutiae in fingerprints and palmprints are extracted automatically by a commercial SDK MegaMatcher $4.0^{2}$.

\subsection{SML vs. SMC}

In this section, we compare the performance of the two methods SML and SMC while using the same parameters for fingerprints and palmprints, i.e., $\sigma=0,\left[\lambda_{l}, \lambda_{h}\right]=$ $[0.08,0.62]$ for SML and $\left[\lambda_{l}, \lambda_{h}\right]=[0.05,0.58]$ for SMC following the settings in [2]. As shown in Figure 1, for both fingerprints and palmprints, the peak genuine scores using SMC are larger than those using SML. Thus, we can conclude that SMC improves the performance when the orientations of minutiae have good quality which is guaranteed by the commercial minutiae extractor MegaMatcher. However, we can also see that the peak genuine scores for palmprints using both SML and SMC are much lower than those for fingerprints.
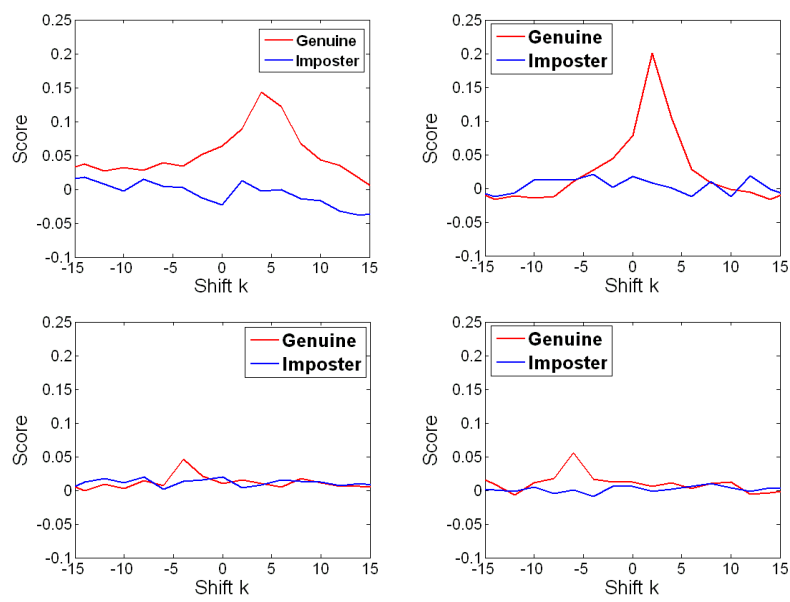

Figure 1. Score distribution between SML (left) and SMC (right) on minutiae sets from fingerprints (up) and palmprints (down).
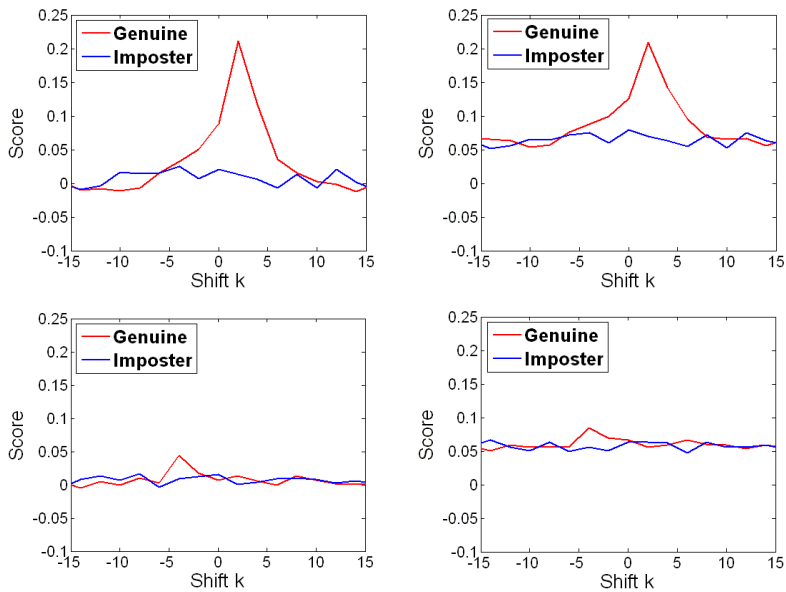

Figure 2. SMC score distribution when using Gaussian pulse on minutiae sets from fingerprints (up) and palmprints (down). $\sigma_{C}=1,\left[\lambda_{l}, \lambda_{h}\right]=[0.05,0.58]$ (left) and $[0.05,1]$ (right).

\subsection{Dirac vs. Gaussian}

In this section, we compare the SMC performance of the two types of pulses Dirac (see Figure 1) and Gaussian we used for minutiae representation. Here we set $\sigma_{C}=1$ for 
Gaussian pulse. From Figure 1 and Figure 2, we can see that the difference between Dirac and Gaussian only appears under large frequency range. Using Gaussian, i.e., $\sigma_{C} \neq 0$, only increases the lower bounds of both genuine scores and imposter scores. It slightly changes the distance between genuine scores and imposter scores. Therefore, we will only set $\sigma=0$ for performance evaluation on THUPALMLAB database in Section 4.

\subsection{Frequency range $\left[\lambda_{l}, \lambda_{h}\right]$}
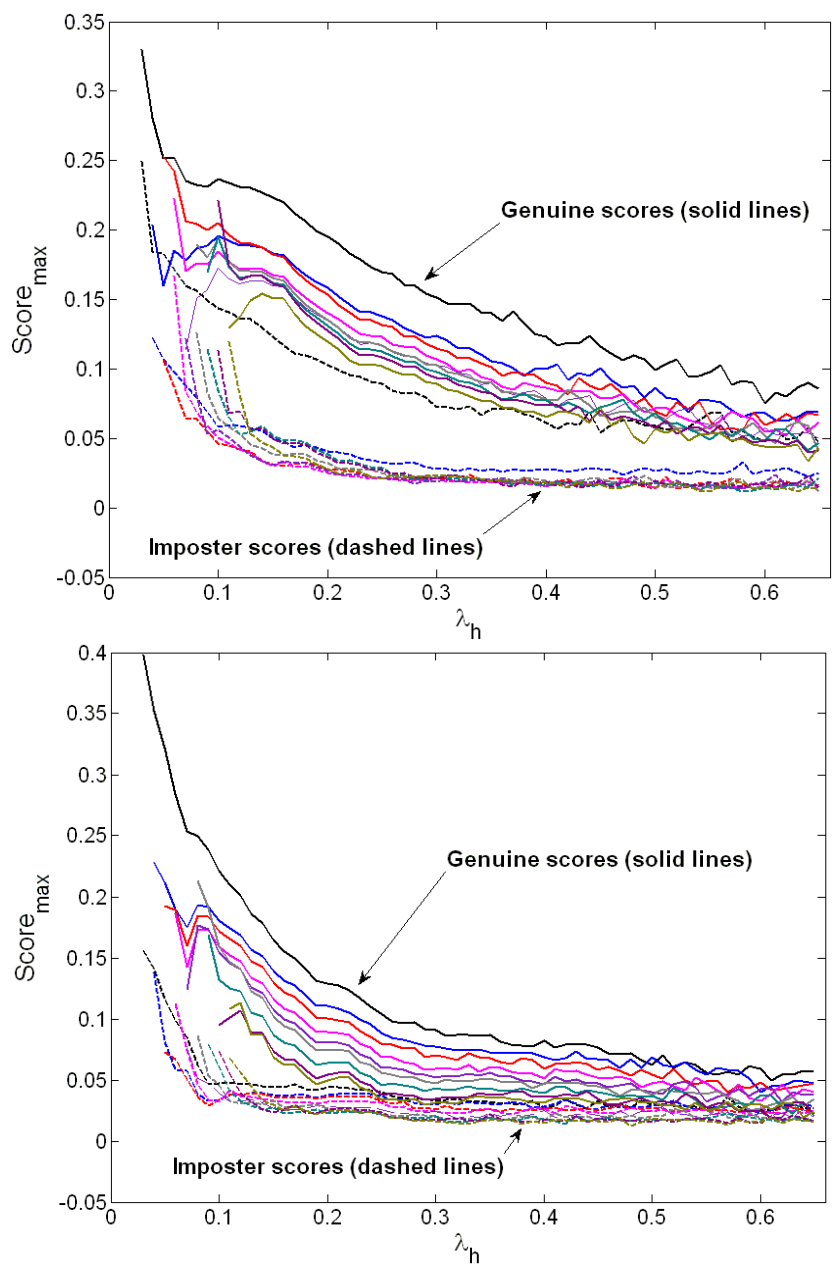

Figure 3. Comparison of maximum score distribution for SML (up) and SMC (down) when $\lambda_{l}=0.02: 0.01: 0.1, \lambda_{h}=\lambda_{l}+0.01: 0.01: 0.65$ for palmprints. The 9 line colors distinguish the 9 values of $\lambda_{l}$ and each pair of a solid line and a dashed line with the same color presents genuine scores and imposter scores.

In this section, we choose the optimal frequency range $\left[\lambda_{l}, \lambda_{h}\right]$ for SML and SMC while varying $\lambda_{l}=[0.2,1]$ and $\lambda_{h}$ $=\left[\lambda_{l}+0.01,0.65\right]$ with a step of 0.01 for palmprints. Again we set $\sigma=0$. In Figure 3, we can see that when $\lambda_{h}$ increases, the distance between the maximum genuine score and imposter score first increases and later decreases until a stable value; when $\lambda_{l}$ increases, the score distance decreases. To achieve better performance, we set the rules to choose the values of $\lambda_{l}$ and $\lambda_{h}$ as following: (1) $\lambda_{h}$ should be larger than the value $\lambda_{h 0}$ when the imposter score is smaller than 0.05 the first time; (2) $\lambda_{l}$ should be the value satisfying a larger genuine score and a smaller imposter score at the chosen value of $\lambda_{h}$. After observing Figure 3 , we choose $\left[\lambda_{l}\right.$, $\left.\lambda_{h}\right]=[0.04,0.3]$ and $[0.05,0.2]$ for SML, and $\left[\lambda_{l}, \lambda_{h}\right]=[0.04$, $0.28]$ and $[0.06,0.16]$ for SMC in palmprints.

\section{EXPERIMENTS AND ANALYSIS}

In this section, we evaluate spectral minutiae based highresolution palmprint matching on a large database and compare the performance with that for fingerprints.

\subsection{Databases}

We obtain the public high-resolution palmprint database THUPALMLAM released by Tsinghua University for evaluation. The database contains 1280 palmprints from 80 subjects (left and right palms of each subject, 8 impressions per palm). These images were captured using a commercial palmprint scanner from Hisign. The image size is $2040 \mathrm{x}$ 2040 pixels, with a resolution of 500 ppi and 256 gray levels. We use the images from the last 50 subjects for palmprint matching evaluation, i.e. $800(=50 \times 2 \times 8)$ images. Then we have 2800 genuine scores and 4950 imposter scores for the subset. In coordinate to [2], we use the results of fingerprint matching [2] on the public fingerprint database $\mathrm{FVC} 2002 \mathrm{DB} 2 \mathrm{~A}$ for performance comparison. The database contains 100 fingers, with 8 images per finger, and only images $1,2,7,8$ for each finger are chosen.

\subsection{Experiments}

Table 2. Matching results on THUPALMLAB database

\begin{tabular}{|c|c|c|c|c|}
\hline \multirow{2}{*}{ Database } & \multirow{2}{*}{ Method } & \multicolumn{2}{|r|}{ Parameters } & \multirow{2}{*}{ EER } \\
\hline & & $\sigma$ & {$\left[\lambda_{l}, \lambda_{h}\right]$} & \\
\hline \multirow{2}{*}{ FVC2002DB2A } & SML & 0 & {$[0.08,0.62]$} & $5.1 \%$ \\
\hline & SMC & 0 & {$[0.05,0.58]$} & $3.05 \%$ \\
\hline \multirow{4}{*}{ THUPALMLAB } & \multirow{2}{*}{ SML } & \multirow{2}{*}{0} & {$[0.04,0.3]$} & $15.89 \%$ \\
\hline & & & {$[0.05,0.2]$} & $16.7 \%$ \\
\hline & \multirow{2}{*}{ SMC } & \multirow{2}{*}{0} & {$[0.04,0.28]$} & $15.58 \%$ \\
\hline & & & {$[0.06,0.16]$} & $14.2 \%$ \\
\hline
\end{tabular}

We evaluate spectral minutiae based matching for palmprints using both SML and SMC methods on THUPALMLAB database and compare with the performance for fingerprints on FVC2002 DB2A. The optimal settings of parameters $\sigma_{L}, \sigma_{C}, \lambda_{1}$ and $\lambda_{\mathrm{h}}$ are decided by following Section 3.3, which are shown together with the matching results in Table 2 and the ROC curves are shown in Figure 4 and Figure 5. EER $15.89 \%$ and $14.2 \%$ are achieved on the palmprint database using SML with $\left[\lambda_{l}, \lambda_{h}\right]$ $=[0.04,0.3]$ and $\mathrm{SMC}$ with $\left[\lambda_{l}, \lambda_{h}\right]=[0.06,0.16]$ respectively. And when using SML, the lager frequency 
range performs better, while the opposite when using SMC. However, compared to fingerprints with EER of $5.1 \%$ for SML and $3.05 \%$ for SMC, the performance of spectral minutiae representation in palmprints is much worse. Furthermore, it is also worse than the performance of a baseline MinutiaCode [4] based system with EER of $10.09 \%$ on the same palmprint database while using the demo software PalmDemo ${ }^{3}$ released by the authors.

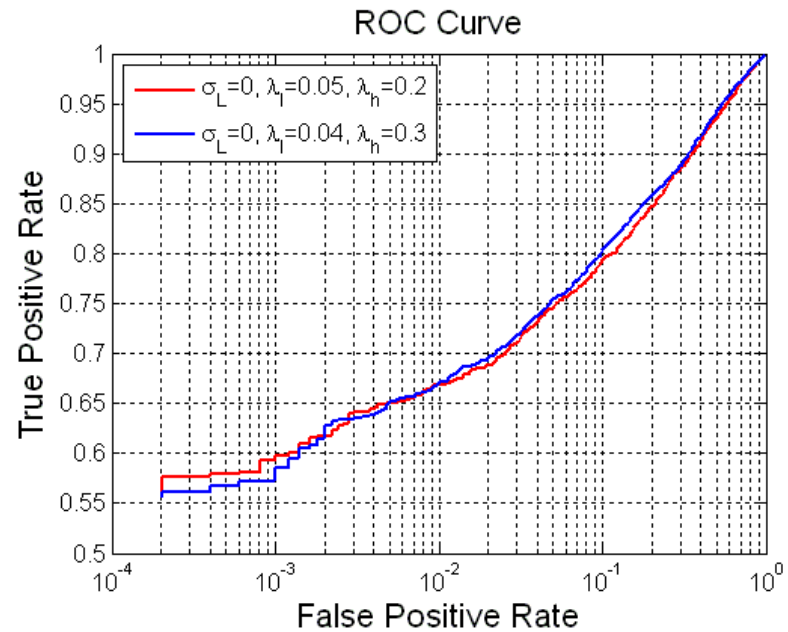

Figure 4. ROC curves using SML when $\left[\lambda_{l}, \lambda_{h}\right]=[0.04,0.3]$ and $[0.05,0.2]$ respectively ROC Curve

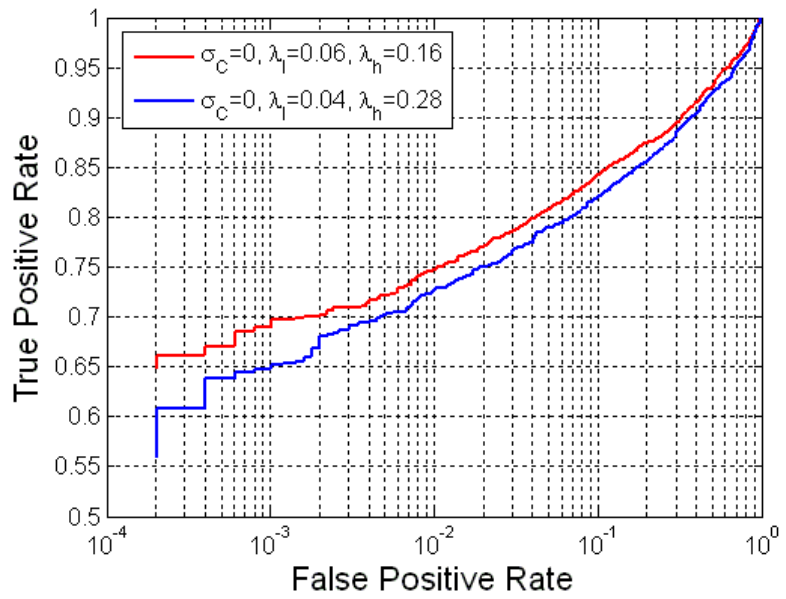

Figure 5. ROC curves using SMC when $\left[\lambda_{l}, \lambda_{h}\right]=[0.04,0.28]$ and $[0.06,0.16]$ respectively

\subsection{Discussion}

When applying spectral minutiae, the only difference between fingerprints and palmprints will be the minutiae feature property. And the feature property is related to the original image. As shown in Figure 6, we can see the palmprint will introduce larger non-linear distortion than the fingerprint due to the larger area of elastic skin in palmprints. Furthermore, after a statistical analysis of minutiae for the palmprint and fingerprint databases as shown in Table 3, we can find that the average number of minutiae in palmprints is much larger and about 30 times of that in fingerprints. It can result in that the spectral minutiae representation method in palmprints is more suffering to spurious minutiae than that in fingerprints.

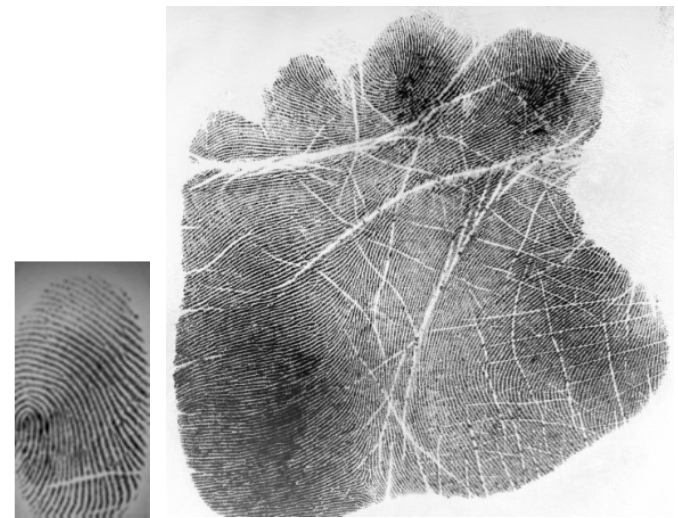

Figure 6. Sample images: a fingerprint and a palmprint

Table 3. Statistics of minutiae in fingerprints and palmprints

\begin{tabular}{c|c}
\hline \hline Database & Avg. Number of Minutiae \\
\hline FVC2002DB2A & 42 \\
\hline THUPALMLAB & 1249 \\
\hline \hline
\end{tabular}

\section{CONCLUSION}

In this work, we applied spectral minutiae representation to palmprints for minutiae-based matching. The direct matching results on the high-resolution palmprint database THUPALMLAB are still much worse than those for fingerprints, i.e., EER 15.89\% for SML and 14.2\% for SMC in palmprints, compared to $5.1 \%$ and $3.05 \%$ in fingerprints. The worse performance mainly due to larger non-linear distortion and larger number of minutiae in palmprints. To improve the performance, we will focus on incorporating minutiae quality information and regional combination.

\section{REFERENCES}

[1] H. Xu, R. Veldhuis, A. Bazen, T. Kevenaar, T. Akkermans, and B. Gokberk, "Fingerprint verification using spectral minutiae representations," Information Forensics and Security, IEEE Transactions on, vol. 4, pp. 397-409, Sept. 2009.

[2] H. Xu and R. N. Veldhuis, "Complex spectral minutiae representation for fingerprint recognition," in Computer Vision and Pattern Recognition Workshops (CVPRW), 2010 IEEE Computer Society Conference on, pp. 1-8, 13-18 2010.

[3] THUPALMLAB Palmprint Database, Tsinghua University. http://ivg.au.tsinghua.edu.cn/index.php?n=Data.Tsinghua500ppi

[4] A. Jain and J. Feng, "Latent palmprint matching," IEEE Trans. Pattern Anal. Mach. Intell., vol. 31, no. 6, pp. 1032-1047, 2009. 